\title{
Optimal Workpiece Orientation to Reduce the Energy Consumption of a Milling Process
}

\author{
Gianni Campatelli ${ }^{1, \#}$, Antonio Scippa', Lorenzo Lorenzini', and Ryuta Sato ${ }^{2}$ \\ 1 Department of Industrial Engineering, University of Florence, Via di S.Marta 3, 50139 Florence, Italy \\ 2 Department of Mechanical Engineering, Kobe University, Kobe, Japan \\ \# Corresponding Author / E-mail: gianni.campatelli@unifi.it, TEL: +39-055-2758728
}

KEYWORDS: Green manufacturing, Parameter optimization, Milling, Workpiece orientation

\begin{abstract}
Energy consumption in the manufacturing sector is becoming a very hot topic due to its significant ecological relevance, especially for energy intensive processes such as machining. Machining finds nowadays large application mainly due to its high performance, in terms of both surface finish and tolerances achievable; as an example this is the key technology in dies and molds production, largely used in the automotive and housewares sectors. Process optimization could be carried out using different strategies, as already proposed by many authors, such as by optimizing machining parameters or implementing alternative toolpath capable of reducing both machining time and energy consumption. Within this paper will be presented a novel approach that takes into account the product orientation within the working zone of the machine. Milling machines are usually non symmetric regarding the energy consumptions of the axes due to the different masses that have to be moved, hence product orientation could sensibly affect energy consumption in performing a toolpath. Optimizing product orientation has the advantage not to affect product quality and require no adjustment of machining parameters. An approach to model the machine power consumption and to optimize the workpiece orientation is presented together with the results of validation experiments.
\end{abstract}

Manuscript received: July 1, 2014 / Revised: November 19, 2014 / Accepted: November 25, 2014

\section{Introduction}

One of the most relevant challenges in modern manufacturing is the reduction of the production energy consumption as far as possible. ${ }^{1}$ With the actual development of economy, global energy demand is estimated to increase by one-third in 2035 comparing with 2010 , therefore to reach 16,750 million tonnes of oil equivalent. ${ }^{2}$ Despite the increasing employment of green technology to produce electric power, the global mix of energy sources is still highly based on fossil fuel; the share was accounting for $81 \%$ in 2008 and the forecast is to reach $74 \%$ in $2035 .^{2}$ The growing demand for fossil fuels led to $\mathrm{CO}_{2}$ emissions rising from 29.3 gigatonnes (Gt) in 2008 to $35.4 \mathrm{Gt}$ in $2035 .{ }^{2}$ Machining is surely one of the most important and widespread processes employed in the manufacturing sector, especially for high quality products that require accurate surface finish and narrow tolerances. In Europe, 2012 data from Europe's Energy Portal, ${ }^{3}$ about $25 \%$ of the primary energy consumption is used by the industries and a margin for improvement of about $30 \%$ has been estimated. Fig. 1 shows the Italian power consumption data by sector in 2010, while Fig. 2 presents a detailed analysis of the industrial sector; the data reported in these figures are available on the website of the Italian energy distributor, TERNA. ${ }^{4}$ From these data the relevant contribution due to machining clearly appears. Also compared to other manufactruing technologies the machining appears as very relevant. ${ }^{5}$ In general the environmental impact of machining processes is due mainly to two sources: the first is related to emission of polluting disposable items, such as coolant and lubricant fluids and cutting inserts, and the second is related to electrical energy consumed by the process itself. Regarding the environmental effects of lubrication it is important to highlight that the traditional flooded lubrication generates an environmental impact, expressed in terms of $\mathrm{kg} \mathrm{CO}_{2}$ equivalent, nearly comparable with the effect of the machining process energy consumption. ${ }^{6}$ This effect is mainly due to the environmental issues related to recycling processes of cutting fluids and the wet scraps treatments. The introduction of more environmental friendly technology, such as dry and near dry technologies, ${ }^{7}$ has increased the share of environmental burden due to the electrical energy consumption. ${ }^{8}$ This is a general consideration that must be contextualized on a regional basis both for the different technological mix used by different nations to produce electrical energy and other local environmental hot issues (i.e., water needs higher than 


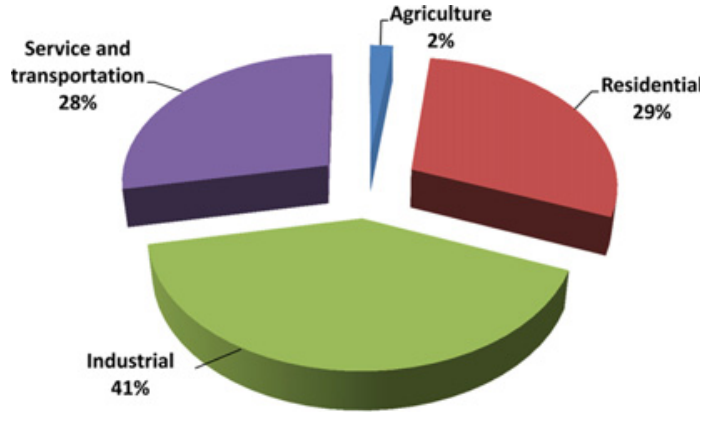

Fig. 1 Power consumption by sector in Italy (year 2010)

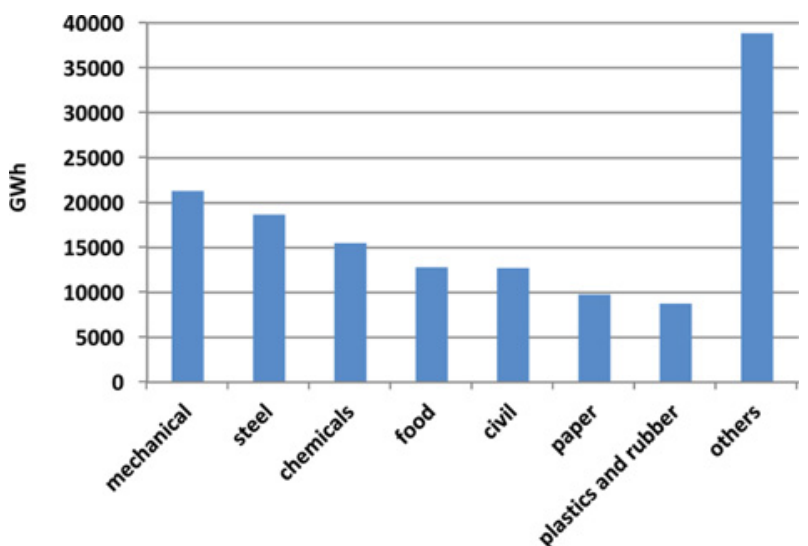

Fig. 2 Power consumption in Italy (year 2010) for the industrial sector in GWh

the sustainable availability of the country).

Also the EU Commission recognized that machine tools play a very important role in this scenario. Their efficiency is so crucial that this type of product has been proposed for inclusion in the product categories regulated by the Ecodesign Directive. ${ }^{9}$ Also, the Kyoto Protocol ${ }^{10}$ reports on the importance of achieving reductions in energy consumption through the development of different design features of machine tools. For this reason, machines equipped with new low power consumptions technologies and integrated power meter monitors are about to reach the market, such as the new machines presented during 2014 by DMG Mori Seiki ${ }^{\circledR}{ }^{11}$ one of the market leaders, that wants to increase the consciousness of the users by integrating a power meter on the front screen of the new Cielo ${ }^{\circledR}$ controller and has decided to equip its machines with high efficiency motors.

In the scientific literature the issues related to energy consumption optimization in milling have been treated with an holistic approach and a plurality of factors have been taken into account. ${ }^{12}$ As cited before, many authors have initially focused their efforts in assessing and minimizing the environmental impact of machining processes considering the employment of alternative cutting fluids or lubrication technologies. ${ }^{13,14}$ In particular, it has been highlighted how the actual cutting fluids are potentially dangerous for health and how the modern solutions should try to be as dry as possible. ${ }^{8}$ One interesting trend is the ever increasing use of minimal quantity lubrication technology (MQL) that entails the employment of a very small quantity of cutting fluid, ${ }^{15}$ maintaining the capability to mitigate the negative effects in terms of surface quality and tool wear of dry machining. Further new technologies are arising in this field, such as the cryogenic cooling that

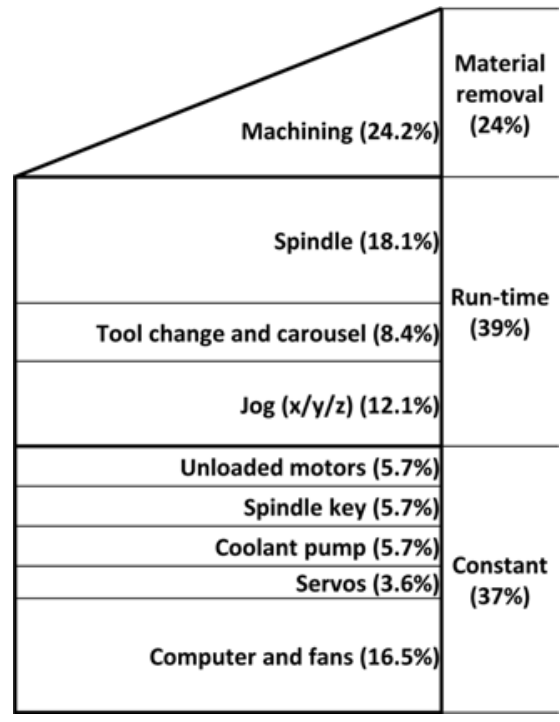

Fig. 3 Analysis of a milling machine energy need (Dahmus, 2007)

uses liquid nitrogen in order to lubricate the process, drastically reducing the environmental impact and also providing side advantages such as the chance to avoid the workpiece final cleaning. ${ }^{16,17}$

Other research activities have been focused on developing methods for optimal cutting parameters selection in order to minimize energy consumption. $^{18}$ Most of the authors have used experimental approaches, such as Response Surface Method ${ }^{19}$ and Taguchi Approach, ${ }^{20}$ in order to define the machining parameters (cutting speed, feed rate, depth of cut and lubrication) that minimize the specific cutting energy. The energy consumption analysis has been carried out along with the machining cost analysis in the model proposed by Anderberg et al. $^{21}$ and the relationship between cutting parameters, machining costs and energy consumption was evaluated in a $\mathrm{CNC}$ machining environment. The energy cost has been also optimized taking into account the cost fluctuation and rescheduling the production accordingly. ${ }^{22,23}$ Also an analysis of the available database to access green manufacturing data has been developed.$^{24}$ For modern machining centres is proved that the energy required by the ancillary systems is usually larger than the one needed by the spindle, some solutions could hence be found to reduce this value. A detailed analysis of the energy required by the machine systems has been carried out by Dahmus ${ }^{25}$ and it highlighted how the ancillary systems energy consumption is generally high; in Fig. 3 is reported an analysis carried out for a Bridgeport milling machine. As stated before, this situation has driven the regulation organizations, like the EU Commission, and manufacturers to develop strategies to improve the efficiency of the machine tools components.

This energy consumption pattern has been proven also for different milling machine tools, ${ }^{26}$ suggesting how it is critical to reduce the power consumption of the machine tool stand-by phase. One interesting proposal comes from Neugebauer et al. ${ }^{27}$ that has shown how the share of ancillary devices and supporting systems in the total energy consumption of the machine tools typically increases with the machine size, mainly due to the higher mass of the machine. This means that a manufacturing company should correctly target the size of new machines considering multiple issues, such as: flexibility to 
produce different workpiece, energy consumption, acquisition cost, quality. In general, a larger machine entails lower power efficiency and quality, but could provide a higher degree of flexibility to the production company. There is also a time dependent trend in the energy needed by a machine tool: the introduction of newer high accuracy machines, with greater complexity and higher moving masses, $\mathrm{s}$ responsible for an increase in the consumption for machining operations, as presented by the works of, ref. 28, 29 both of which studied the same Mori Seiki three-axis NV1500DCG machine. This trend is confirmed also for five-axis machines, where generally the weight of the structure is heavier than three-axis machines, due to the presence of additional motors and support systems. ${ }^{30}$ On the other hand, simpler machines, like lathes, have a lower share of energy needed by the ancillary systems respect to the one used by the spindle: for a modern $\mathrm{CNC}$ lathe the percentage of energy used by the spindle is about $61-69 \%$ respect to the total energy. ${ }^{31}$

Another crucial issue regarding the machining process optimization is due to the effect of green strategies, mainly dry lubrication, on the surface finish of the product. ${ }^{32}$ This means that green strategies could not be applied without any constraints, but their application requires some preliminary study taking into account the optimal compromise between quality, production, service cost and obviously environmental impact. One general solution is to stress the reduction of environmental impact on the roughing phase of the workpiece, where the surface finish is not critical and dry lubrication could be used without relevant drawbacks, using hence a setup with a lower environmental efficiency for the finishing operations.

In order to optimize the energy requirement of a machine tool for a specific task a reliable model of the power needed by a machine is required. These models are mainly based on experimental tests aimed at characterizing the energy consumption of the machine. Recent studies have been carried out to develop analytical models of machine tool components, such as the analytical study of the feed drive carried out by ref. 33, in order to improve the predictive capability of the model. However, this model requires that each component of the system would be characterized and this is feasible in a research environment only, while it could hardly find application in a manufacturing company. Moreover, it is important to consider that the axis characteristic could change over time due to the wear of the components that usually increase the energy need for a specific task. To solve these issues a fast experimental approach to characterize the axis power consumption is required. In the next paragraph the authors introduce a simple model that could be setup performing simple axis movement and using an energy monitoring system. The general idea is to use such model to optimize the axis energy consumption for a specific toolpath. The authors have developed an approach that could reduce the energy consumption of the process without the need of drastic change on the process, as required by most of the state-of-theart solution proposed since. The advantage is minor respect to other solutions but the approach does not require any installation and does not affect quality or cost. The idea is to change the workpiece orientation in order to reduce the energy consumption taking advantage of the asymmetry of most milling machine. This strategy could provide a lower reduction of energy consumption compared with more drastic solutions, such as the change of lubrication strategy, but has two main advantages: this reduction could be added to the one already obtained with previous activities (i.e., optimal lubrication selection) and could be achieved without requiring additional investments. The only effort required is to find the optimal orientation of the workpiece on the machine table. The feasibility of this strategy has been highlighted also by Kong ${ }^{34}$ that studied the effect of maximum allowable feed respect to different axis orientation, due to the different masses of the axis and the maximum torque of the motors. However, for modern machines that are usually characterized by high allowable accelerations it is unusual that the programmed toolpath would require axis accelerations higher than the machine capabilities. This is an important issue for the machining time because when the acceleration required is greater than the allowable one the machining time increases. This could happen when the toolpath is really "spiky and have great acceleration near the turning points, like for a manually programmed zigzag toolpath. In this case the motors cannot provide the acceleration required to maintain the feed constant (like in case of a sharp turn), so the feed is reduced and the cycle time increases. In case of fluent toolpaths the acceleration are usually lower thanks to the smoothing of the profile and so the machine is able to maintain a constant feed. Actually, most of the CAM software are pushing for obtaining fluent toolpaths also for roughing operations because this approach provides other side advantages in terms of vibration and surface quality. In general, for fluent toolpaths is possible to state that the cycle time is not affected by the workpiece orientation, since accelerations are lower than the motor capabilities, as proved by the experimental tests carried out by the authors. However, the workpiece orientation affects the acceleration required by each axis along the toolpath, hence influencing the energy consumption. By the energy consumption point of view, the optimal workpiece orientation is hence the one that allows moving the largest share of acceleration on the most efficient axis.

\section{Characterization of the Machine Axes Consumption}

The machine tool used for the experimental activity is a high performance five-axis milling machine produced by Mori Seiki, the NMV1500DCG loaned to the authors by MTTRF for research activities, whose architecture is reported in Fig. 4. This machine is equipped with a high-speed spindle that could reach 40,000 rpm and has a maximum power of $5.5 \mathrm{~kW}$. The working space is limited to $420 \mathrm{~mm} \times 210 \mathrm{~mm} \times 400 \mathrm{~mm}$ respectively in the $\mathrm{x}, \mathrm{y}$ and $\mathrm{z}$ direction. The architecture of this machine, common to many others, is responsible for different axis inertia and energy consumption due to the different mass of the moving parts. For example, the movement of $z$ axis involves the vertical octagon only, while a movement of the $\mathrm{x}$ axis involves the support for the $\mathrm{x}$ slider and the vertical octagon. A movement of the $y$ axis involves a slider that support all the others. This non symmetric behaviour of the studied machine tool is due to the specific machine architecture, but is also a general feature of most milling machine, due to the positioning of the spindle on at least a couple of moving axes. Only the parallel kinematic machines have perfectly equal axis behaviour. In particular, for the studied machine it is noticeable that the y axis has greater masses to be moved and it has a different actuation strategy. For this axis two coupled motors are 


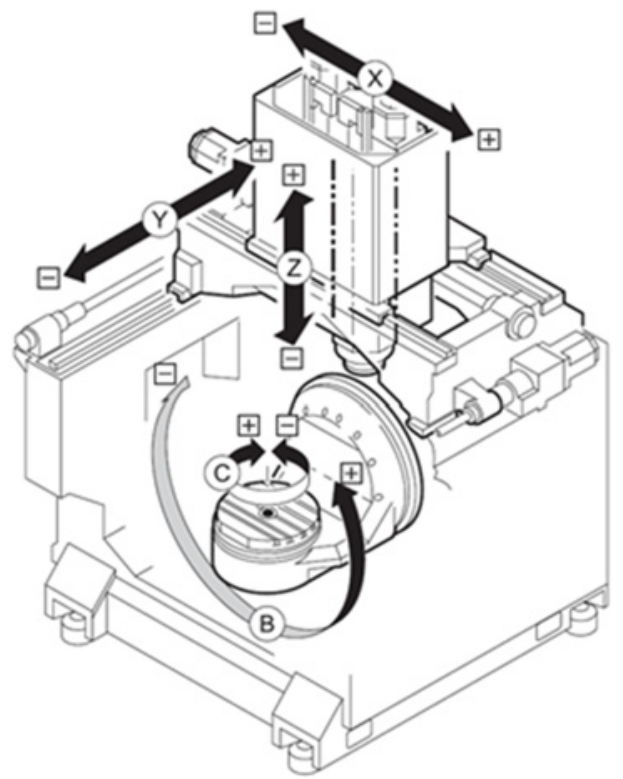

Fig. 4 Kinematic scheme of NMV 1500 DCG milling center

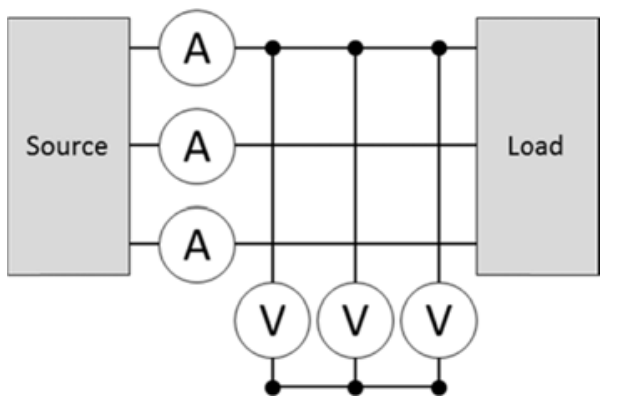

Fig. 5 Architecture of the electrical power measurement system

used, instead of the single one used for the $\mathrm{x}$ axis. So also the mechanical link and the electrical system could have different efficiencies.

In order to develop a model for the machine consumption the first step has been the experimental characterization of the axes energy consumption. During each test the instantaneous electrical power demanded by the machine tool has been acquired using three current gauges produced by LEM (LEM AT 10 B420L, hall effect sensor with open core and a range $0-10 \mathrm{~A}$ ) whose signals were acquired together with the phase voltage with a National Instruments simultaneous sampling system, equipped with NI-9215 cards, at the frequency of 100 $\mathrm{Hz} /$ channel.

The choice to use three pairs of voltage/current measurement probes, mounted on the system has shown in Fig. 5, has been considered in order to measure the power for each phase. It is so irrelevant the configuration of the machine, that could be wye or delta, as presented by ref. 35 .

The signals were post-processed using a Matlab ${ }^{\circledR}$ routine in order to acquire the instantaneous power during each test. The post-processing routine computes the instantaneous power demanded by each of the three phases of the main machine to electrical grid connection, based on the current and voltage sensors data. The power required by the grid was chosen instead of the power flow through the numerical control because this measurement includes also the efficiency of the AC/DC

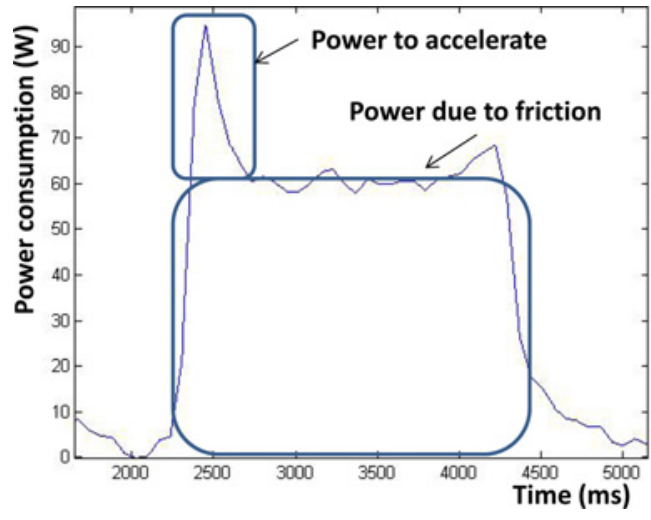

Fig. 6 Axis test power consumption

converters and the numerical control itself. The machine has been actuated in order to have an initial stand-by phase before the experimental test. The post-processing algorithm uses this period to assess the machine stand-by power in order to subtract this value to the acquired one to find the net power requirement of the axis. For further validation, also the power flow through the direct link of numerical control has been measured using a DC current meter by LEM (HTR 50-SB, the direct link has been substituted with a cable). In this case the constant contribution due to the continuous activation of the $\mathrm{z}$ axis has been removed: this axis is powered up during the machining and must sustain the weight of the axis and the spindle. The second acquired value is lower than the one computed at the grid connection but it is perfectly proportional to it. The difference is mainly due to the efficiency of the AC/DC converter.

The experimental strategy to assess the axes power consumption is based on moving each axis independently at different speed for a standard length. The different speeds are needed to model the effect of speed on the efficiency of all the moving system, that include all the components, starting from the power electronics to the mechanical prismatic joints. A typical resulting graph of the tests is the one reported in Fig. 6, where both the power required for the initial axis acceleration and final deceleration, respectively the initial and final peaks of power, and the constant power required to overcome friction of the slider and the mechanism efficiency are noticeable.

For each axis 10 different speeds have been tested, ranging from $1,500 \mathrm{~mm} / \mathrm{min}$ to $6,000 \mathrm{~mm} / \mathrm{min}$, for lower speed acquired data could not be reliable because the power is relatively low and the signal has an excessive Signal to Noise ratio. The travelled length for each axis has been fixed to $200 \mathrm{~mm}$, near the maximum range of the most limited axis. This value allows a long acquisition time, hence reducing the errors due to the possible signal noise and enabling the axis to reach the imposed feed.

Thanks to these tests the power consumption of each axis has been characterized, allowing the development of a model. The model has been chosen as simple as possible and takes into account both the acceleration and efficiency of the axis system by means of two coefficients. These are respectively an equivalent moving mass of the axis, that is responsible for the power need to accelerate the axis, and an equivalent friction coefficient, that takes into account the efficiency of the system, both electrical and mechanical. These two coefficients are equivalent values so could not have a real reference. As an 
Table 1 Equivalent coefficients of axis power consumption

\begin{tabular}{ccc}
\hline Axis & Equivalent mass $(\mathrm{kg})$ & Equivalent friction \\
\hline $\mathrm{X}$ & 768.7 & 0.061 \\
\hline $\mathrm{Y}$ & 1123.4 & 0.042 \\
\hline $\mathrm{Z}$ & 164.7 & 0.469 \\
\hline
\end{tabular}

example, the equivalent friction is not representative of the real mechanical friction in the prismatic joint but its definition is suitable to provide a general measure of the axis system. The equivalent mass usually provides a value that is comparable to the axis moving masses as obtainable by the CAD files of the machine, available thanks to Mori Seiki ${ }^{\circledR}$ DTL (Digital Technology Laboratory). In general, the obtained value is higher due to the presence of ancillary and auxiliary systems that are not reported in the structural CAD file of the machine. The model proposed to predict the energy consumption (E) of an axis movement could be expressed with the following formula (1).

$$
E=\int_{0}^{S}\left[\left(M x \cdot a_{x}(s)+\mu_{x} \cdot M x \cdot g\right)+\left(M y \cdot a_{y}(s)+\mu_{y} \cdot M y \cdot g\right)\right] d s
$$

Where $M x$ and $M y$ are the equivalent masses of the axes respectively, $a_{x}(\mathrm{~s})$ and $a_{y}(\mathrm{~s})$ the instantaneous accelerations, $\mu_{x}$ and $\mu_{y}$ the equivalent friction coefficients, $s$ the length of the toolpath and $g$ the gravity acceleration. The parameters of the model obtained by the experimental tests for the linear axes are reported in Table 1.

In order to use the proposed model for the evaluation of the machine power need it is necessary to obtain the instantaneous acceleration along the path for a general toolpath. These data are obtained thanks to a model of the $\mathrm{CNC}$, based on jerk control strategy, that has been developed. Adopting a jerk control strategy the developed CNC model uses a bell shaped time-position profile in order to accurately represent the real behaviour of a modern $\mathrm{CNC}$ as much as possible. To setup this model is necessary to obtain the values of the maximum accelerations and jerks for all the axis. Since these values do not depend only on the motors but also on the masses and mechanical link of the axis, the choice has been to evaluate them using experimental tests. For a less accurate model also the data provided by the machine manufacturer could be used, anyway it must be considered that these values could change for each machine, due to tolerances of the components, and over time, due to components wear. The developed experimental tests have been carried out measuring the tooltip position during a linear movement along a single axes using a high precision laser interferometer by Renishaw ${ }^{\circledR}$ and post processing the data using a Matlab $^{\circledR}$ routine. From these data the maximum acceleration and jerk of the axis can be obtained.

Such model is capable of predicting the energy consumption of each axis for a general toolpath and has been used for the following workpiece orientation optimization. The model has been validated by performing simple operations, such as linear and circular toolpaths.

\section{Optimal Workpiece Orientation}

Many contributions must be considered in order to compute the power required by a machine tool to perform a given operation (i.e., spindle power, ancillary systems power, axes power). The first contribution is independent from the toolpath orientation because it is mainly related to the workpiece material and the tool engagement (depth of cut and feed per tooth). The second contribution has a dependency on the toolpath selection because different toolpaths are responsible for different machining time, as presented by Rangarajan and Dornfeld. ${ }^{36}$ This aspect gets especially relevant when the toolpath requires rapid accelerations, in which the feed is limited by the maximum acceleration available for the single axis, such as happens in a change of direction when machining a square pocket with morphing strategy. This phenomenon gets less important when a "fluent" toolpath is programmed into the machine, since the acceleration required along the toolpath in order to maintain a constant feed does not exceed axes capabilities. This usually happens in case of finishing operations. The idea of this paper is to optimize the energy consumption of these operations, for which the toolpath is usually imposed by surface requirements and changing the product orientation on the worktable does not sensibly affect machining time. Workpiece orientation influences the machine energy consumption because the axes have different energy consumptions, mainly due to the different masses, and the share of acceleration that each axis must sustain changes with the toolpath rotation. This optimization is interesting because, in case of fluent toolpath, it does not affect the machining time and the related ancillary system power consumption and does not either affect surface quality and accuracy. The quality and accuracy of a workpiece is mainly related to the stiffness of the tooltip along the cutting force direction. Small machines are usually symmetrical due to the reduced stroke of the axis but larger machines could have a different behavior for the $\mathrm{x}$ and $\mathrm{y}$ axis. This could be an issue when a bulky tool is used because the least stiff part of the system will be the axis. In case of finishing operations the tool used is generally slender and become often the least stiff part of the system, so the behavior of the machine in term of quality along different direction is equal due to the axial symmetry of the tool. So as long as the approach is applied for finishing operations with fluent toolpaths, the orientation of the workpiece will not affect quality.

The optimization of the workpiece rotation implies computing the acceleration that a milling machine must provide in order to maintain a constant feed along the defined toolpath. This acceleration is orthogonal to the toolpath and the related energy need could be computed using formula (1). Effects of friction/efficiency of the moving parts of the mechanical chain must be included in this contribution; both data are available in the proposed equivalent axis consumption model. The effect of the different behavior of machine's axis is more evident when high speeds are used, because of the related higher inertia forces. The International motoGP motorbike track of Mugello has been chosen as case study, in order to have a fluent and high speed toolpath. The coordinates of the track have been acquired as splines and the dimensions scaled in accordance with the machine table. This toolpath has been chosen because it represents a fluent toolpath that could be used as an example of a general finishing toolpath, still maintaining an high degree of simplicity. In Fig. 7 the circuit path and an example of two rotations around its center of gravity are reported. The toolpath energy consumption has been computed for different rotation angles and lately tested experimentally to validate the model.

For each rotated toolpath the acceleration along the path can be 

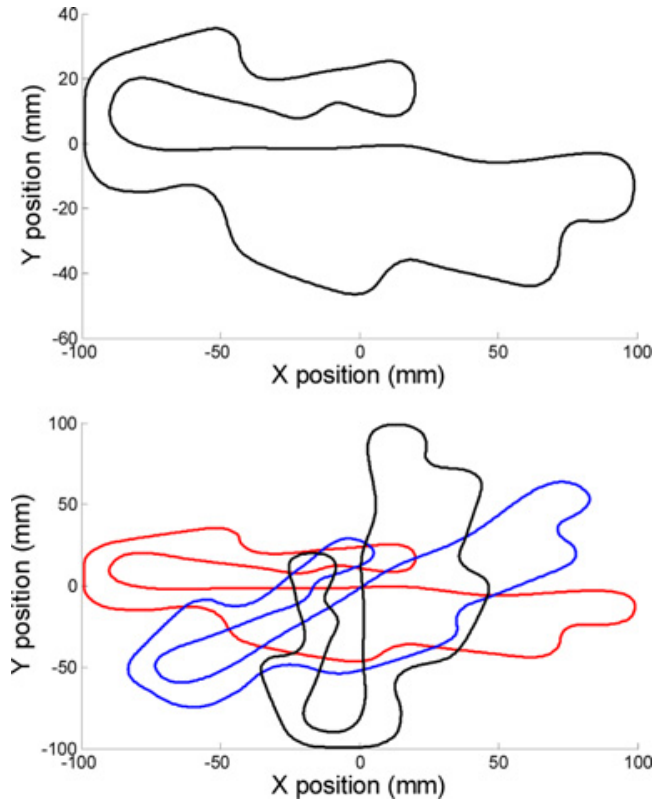

Fig. 7 Mugello toolpath and rotations
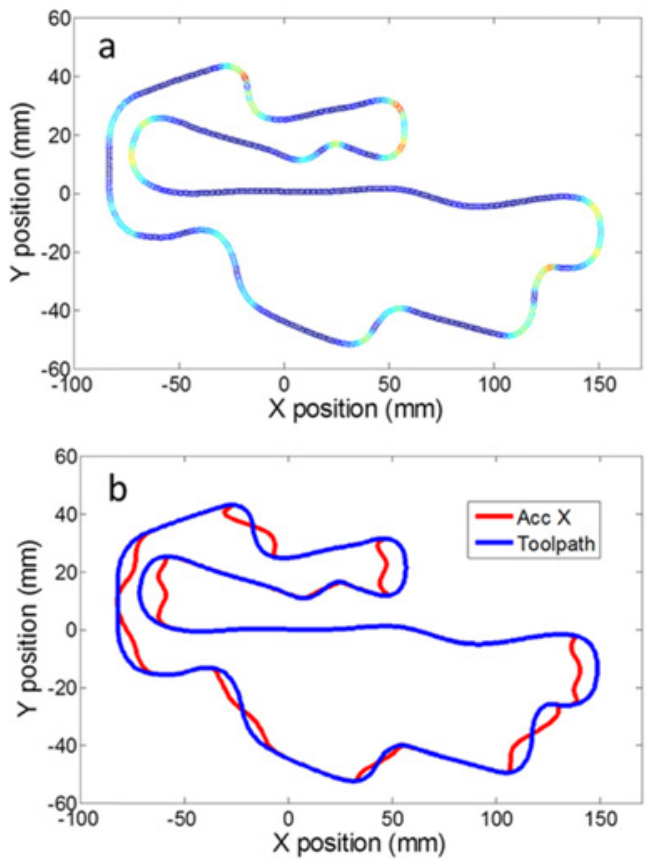

Fig. 8 Toolpath and modulus of acceleration (a) and $\mathrm{x}$ component of acceleration (b)

computed, imposing the feed value, and decomposed along the two axis in order to feed the model (1) to estimate the energy consumption of the machine. In Fig. 8 toolpath with the acceleration modulus, represented using a colormap, and the component of the acceleration along the $\mathrm{x}$ axis are reported; higher values are found when the red line get farther from the nominal profile.

\subsection{Experimental Tests}

The machining operations have been carried out using a Mori Seiki NMV1500 DGC milling centre equipped with a 40,000 rpm spindle. For the specific tests no tool has been mounted and the operation has been carried out in air cutting mode: this test has the aim to evaluate the axis energy consumption only, without considering the cutting

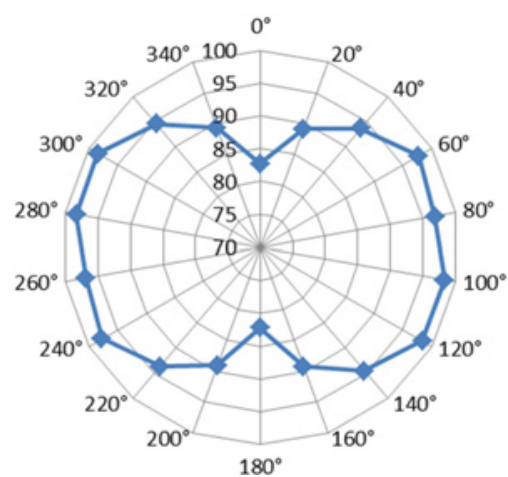

Fig. 9 Power consumption for different toolpath rotation (mean W value)

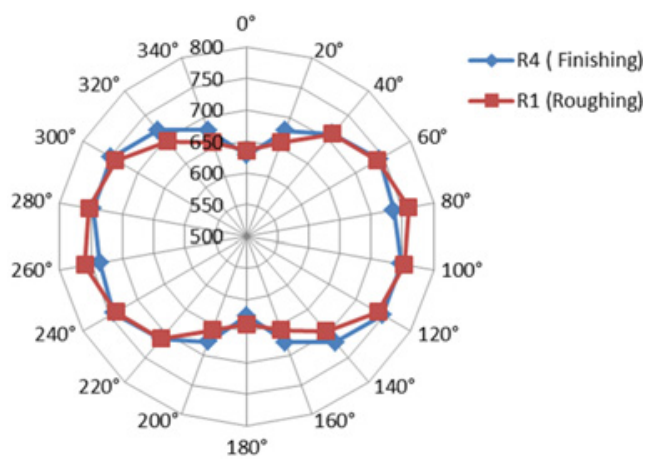

Fig. 10 Power consumption for rotated toolpath in different cutting mode (mean $\mathrm{W}$ value)

energy. The rotation of the workpiece does not affect the cutting energy because the cutting parameters remain the same, as well as the relative position between the workpiece and the tool. This implies that also small variations of the cutting energy due to material non-uniformity do not affect this optimization because each alternative rotated toolpath will sustain exactly the same fluctuation of cutting energy.

From the axis characterization experimental tests has emerged that the machine has an asymmetric behaviour and so it is expected a measurable effect on the energy consumed with different workpiece orientation. In Fig. 9 the experimental results of the Mugello toolpath acquired applying a rotation with a step of $20^{\circ}$ are reported. As obtainable from the graph the behaviours of $\mathrm{x}$ and $\mathrm{y}$ axes are symmetric regarding the acceleration direction so there is no need to rotate the toolpath of $360^{\circ}$ to experimentally characterize the power consumption but a rotation of $180^{\circ}$ is enough. The resulting graph has a lobe geometry that highlights how the toolpath rotation could produce a minimum and a maximum of the axes movement energy. The tests have been carried out using different feeds and all have been performed with air cutting. The resulting energy is relative only to the axes movements, all the other contributions, such as spindle and ancillary systems have not been considered. The experimental machining time is the same in every test and it is equal to $7.6 \mathrm{~s}$ using a feed of $4,000 \mathrm{~mm} / \mathrm{min}$.

Considering that the axis inertia plays a relevant role in the axis power consumption it has been evaluated how the machine power consumption is influenced by the change of axis accelerations. The machine used for the experimental tests has the possibility to change the maximum axis acceleration using different cutting modes. With the G332 command of the FANUC NC the machine could be set from a coarse cutting mode (R1) to a fine cutting mode (R4). In the first case 
the machine has greater accelerations, hence introducing larger errors in the finished product due to the deformation induced by the inertia forces. This cutting mode enlarges also the tolerance between the theoretical and NC programmed toolpath in order to have a smoother profile; this setup is advised for roughing operation. The opposite cutting mode, R4, is the choice that imposes smaller axis accelerations and it is advised for finishing operations: the tolerance range is reduced respect to R1 mode. In this case the machining operations could be longer than in R1 mode but the workpiece final accuracy is obviously greater thank to lower and more gradual inertia forces that excite the machine tool structure. Two intermediate setup, R2 and R3, are also available; these are a compromise of the two previous configurations. In order to evaluate the effects of the cutting modes the experimental tests have been carried out both using R1 and R4 cutting mode. In Fig. 10 the results of these experimental tests are reported, from which it is possible to evaluate how the power needs are very similar for the two configurations. Using R4 configuration, the possible maximum accelerations are higher but the profile is smoother thanks to the enlargement of the tolerances, resulting in a value really close to the R1 mode apart from some specific rotation angles, like $20^{\circ}$ and $160^{\circ}$. Also the time to perform the cycle is nearly the same. Apart small differences, should be highlighted that the position of the maximum and minimum power consumption has not altered by this parameter.

\subsection{Comparison with Simulated Data}

The experimental tests have been compared with the analytical model of the axes energy consumption to validate the proposed model. The tests have been carried out at different speeds, but only at higher feeds the reduction of the energy consumed assumes a relevant entity from the optimization point of view. Due to the increase of the acceleration the energy needed to move the axes become important when the feed is very high, such as in the case of High Speed Cutting; with traditional cutting parameters, especially feed, the axes acceleration consumption is less relevant. In Table 2 the comparison between the predicted and measured energy for high feed cutting, such as with 4,000 and $6,000 \mathrm{~mm} / \mathrm{min}$, is reported highlighting the maximum and minimum values. The optimal rotation angle has been also computed using the analytical approach and it has been successively tested experimentally. The difference between the predicted and measured energy consumption is around $10 \%$ while for the optimal positioning a maximum error of $5^{\circ}$ has been observed.

This difference is probably due to the simple friction model adopted which uses equivalent coefficients that are speed independent. More complex models could be adopted, but it is important to notice that from the production point of view the most interesting result it is not the predicted energy saving, but the optimal rotation angle of the workpiece on the machine table.

From the experimental and simulated analysis has emerged that the axis energy consumption could be reduced by $23 \%$ for this toolpath, with a total energy saving of about $200 \mathrm{~J}$. The result is dependent on the feed chosen and it increases accordingly with this value, it is also strongly related to the geometry and length of the toolpath. Should be reminded that this saving is related only to the energy needed for the axis movement. The reduction of other energy sinks, such as ancillary systems or cutting energy, must be carried out with different strategies;
Table 2 Comparison of analytical and experimental data

\begin{tabular}{ccccc}
\hline feed & \multicolumn{2}{c}{$4000 \mathrm{~mm} / \mathrm{min}$} & \multicolumn{2}{c}{$6000 \mathrm{~mm} / \mathrm{min}$} \\
\hline & Simulated & Exp. & Simulated & Exp. \\
\hline Max energy $(\mathrm{J})$ & 844.2 & 813.5 & 1032.7 & 924.6 \\
\hline Min energy $(\mathrm{J})$ & 672.0 & 623.7 & 805.0 & 709.1 \\
\hline Error max. & $+3.6 \%$ & $+10.5 \%$ \\
\hline Error min & $+7.2 \%$ & $+11.9 \%$ \\
\hline Saving & $215.5(-23 \%)$ & $189.8(-23 \%)$ \\
\hline
\end{tabular}

for example implementing a new activation strategy of the machine components or using different lubrication approaches.

Compared to other energy consumption strategies the proposed one has the advantage to be easily applicable and with no added cost. Moreover, it does not need to update any other machining parameters. For an analysis of the absolute reduction of the energy need some previous studies can be considered ref. 19, 25. Those works assess how the share of energy needed by the axis movement for a milling process is nearly $15 \%$ of the total energy: a saving of about $23 \%$ on axis movement could provide a $3-4 \%$ total energy saving. Although this saving could be considered small, it is necessary to remind that this result is obtained with no relevant changes to the machining process, such as parameters changes or different tooling/lubrication. This result could be obtained nearly for free, just selecting a different workpiece orientation on the machine table. This has also the advantage not to alter the quality and productivity of the process. In order to obtain such a saving the only activity required to the process planner is to characterize once the machine axis power consumption and to perform a simple optimization of the workpiece rotation. The saving percentage could be also higher for material that requires high cutting forces or for which the cutting force are dependent on other parameters, such as cutting speed. ${ }^{37}$ Moreover, this approach could ensure a higher percentage of saving when the impact of spindle and axis movement would become higher, thanks to the introduction of more efficient ancillary systems.

\section{Conclusions}

In this paper a novel approach to reduce the energy consumption for a milling process based on the optimal orientation of the workpiece on the machine table has been presented. Although drastic solutions, such as the elimination of lubrication or the introduction of high efficiency spindles and motors, could have a more relevant impact on the energy consumption of the process the proposed approach has the advantage to require no investment and no adjustment of the other machining parameters.

The approach is intended for finishing operations that use fluent toolpaths, for which the different orientation of the workpiece do not affect cycle time nor quality. In case of roughing operations on large machines the orientation could affect the quality of the workpiece, limiting the possibility for an optimization using the proposed approach.

The energy saving obtainable using this approach is strongly dependent on the toolpath, workpiece material and feed. The saving obtained with the analyzed case study, the Mugello motoGP motorway, is $23 \%$ of the axis energy consumption and, in terms of total energy 
need, could reach the estimated reduction value of $3-4 \%$. In order to perform this optimization is required to model the energy consumption of the axis. The proposed model is based on the use of two coefficients for each axis: an equivalent mass and an equivalent friction coefficient. This model has proven its functionality and it has been validated thanks to experimental tests. The proposed approach can be easily implemented within a numerical code.

\section{ACKNOWLEDGEMENT}

The authors wish to thank the Machine Tool Technology Research Foundation (MTTRF) for providing the machine tool for the tests and for the general support of their research activities. Special thanks are reserved for the staff of Mori Seiki for all the support provided during the installation and use of the machine.

\section{REFERENCES}

1. Dornfeld, D. A., "Moving Towards Green and Sustainable Manufacturing," Int. J. Precis. Eng. Manuf.-Green Tech., Vol. 1, No. 1, pp. 63-66, 2014.

2. International Energy Agency, "World Energy Outlook 2010," www.worldenergyoutlook.org/media/weo2010.pdf (Accessed 10 December 2014)

3. Europe's Energy Portal, "Energy Prices Report," www.energy.eu (Accessed 10 December 2014)

4. TERNA, "Fabbisogno Energetico Nazionale," www.terna.it (Accessed 10 December 2014)

5. Yoon, H.-S., Lee, J.-Y., Kim, H.-S., Kim, M.-S., Kim, E.-S., et al., "A Comparison of Energy Consumption in Bulk Forming, Subtractive, and Additive Processes: Review and Case Study," Int. J. Precis. Eng. Manuf.-Green Tech., Vol. 1, No. 3, pp. 261-279, 2014.

6. Campatelli, G., "Analysis of the Environmental Impact for a Turning Operation of AISI 1040 Steel," Proc. of the PROMS Conference, pp. 6-17, 2009.

7. Weinert, K., Inasaki, I., Sutherland, J., and Wakabayashi, T., "Dry Machining and Minimum Quantity Lubrication," CIRP AnnalsManufacturing Technology, Vol. 53, No. 2, pp. 511-537, 2004.

8. Dahmus, J. B. and Gutowski, T. G., "An Environmental Analysis of Machining," Proc. of the ASME in International Mechanical Engineering Congress and Exposition, pp. 643-652, 2004.

9. EPTA, "Study for Preparing the First Working Plan of the Eco-Design Directive," http://ec.europa.eu/enterprise/policies/sustainable-business/ files/workingplan_finalreport_en.pdf (Accessed 10 December 2014)

10. United Nations, "United Nations Framework Convention on Climate Change," http://www.globaldialoguefoundation.org/files/ENV.2009jun.unframeworkconventionclimate.pdf (Accessed 10 December 2014)
11. DMG Mori Seiki, "DMG Energy Saving," http://en.dmgmori.com/ dmg-energy-saving/dmg-energy-saving (Accessed 10 December 2014)

12. Yun, J.-H., Jeong, M.-S., Lee, S.-K., Jeon, J.-W., Park, J.-Y., et al., "Sustainable Production of Helical Pinion Gears: Environmental Effects and Product Quality," Int. J. Precis. Eng. Manuf.-Green Tech., Vol. 1, No. 1, pp. 37-41, 2014.

13. Davoodi, B. and Tazehkandi, A. H., "Experimental Investigation and Optimization of Cutting Parameters in Dry and Wet Machining of Aluminum Alloy 5083 in order to Remove Cutting Fluid," Journal of Cleaner Production, Vol. 68, pp. 234-242, 2014.

14. Lawal, S. A., Choudhury, I. A., and Nukman, Y., "A Critical Assessment of Lubrication Techniques in Machining Processes: A Case for Minimum Quantity Lubrication using Vegetable Oil-Based Lubricant," Journal of Cleaner Production, Vol. 41, pp. 210-221, 2013.

15. Gaitonde, V., Karnik, S., and Davim, J. P., "Selection of Optimal MQL and Cutting Conditions for Enhancing Machinability in Turning of Brass," Journal of Materials Processing Technology, Vol. 204, No. 1, pp. 459-464, 2008.

16. Pusavec, F., Krajnik, P., and Kopac, J., "Transitioning to Sustainable Production-Part I: Application on Machining Technologies," Journal of Cleaner Production, Vol. 18, No. 2, pp. 174-184, 2010.

17. Pusavec, F., Kramar, D., Krajnik, P., and Kopac, J., "Transitioning to Sustainable Production-Part II: Evaluation of Sustainable Machining Technologies," Journal of Cleaner Production, Vol. 18, No. 12, pp. 1211-1221, 2010.

18. Lee, W., Lee, C.-Y., and Min, B.-K., "Simulation-Based Energy Usage Profiling of Machine Tool at the Component Level," Int. J. Precis. Eng. Manuf.-Green Tech., Vol. 1, No. 3, pp. 183-189, 2014.

19. Campatelli, G., Lorenzini, L., and Scippa, A., "Optimization of Process Parameters using a Response Surface Method for Minimizing Power Consumption in the Milling of Carbon Steel," Journal of Cleaner Production, Vol. 66, pp. 309-316, 2014.

20. Fratila, D. and Caizar, C., "Application of Taguchi Method to Selection of Optimal Lubrication and Cutting Conditions in Face Milling Of Almg 3 ," Journal of Cleaner Production, Vol. 19, No. 6, pp. 640-645, 2011.

21. Anderberg, S., Kara, S., and Beno, T., "Impact of Energy Efficiency on Computer Numerically Controlled Machining," Proc. of the Institution of Mechanical Engineers, Part B: Journal of Engineering Manufacture, Vol. 224, No. 4, pp. 531-541, 2010.

22. Shrouf, F., Ordieres-Meré, J., García-Sánchez, A., and Ortega-Mier, M., "Optimizing the Production Scheduling of a Single Machine to Minimize Total Energy Consumption Costs," Journal of Cleaner Production, Vol. 67, pp. 197-207, 2014.

23. Jo, H., Noh, S. D., and Cho, Y., "An Agile Operations Management System for Green Factory,” Int. J. Precis. Eng. Manuf.-Green Tech., Vol. 1, No. 2, pp. 131-143, 2014. 
24. Ahn, S.-H., "An Evaluation of Green Manufacturing Technologies based on Research Databases," Int. J. Precis. Eng. Manuf.-Green Tech., Vol. 1, No. 1, pp. 5-9, 2014.

25. Dahmus, J., "Applications of Industrial Ecology: Manufacturing, Recycling, and Efficiency," Ph.D. Thesis, Department of Mechanical Engineering, Massachusetts Institute of Technology, 2007.

26. Diaz, N., Redelsheimer, E., and Dornfeld, D., "Energy Consumption Characterization and Reduction Strategies for Milling Machine Tool Use," in: Glocalized Solutions for Sustainability in Manufacturing, Hesselbach, J. and Herrmann, C., (Eds.), Springer Berlin Heidelberg, pp. 263-267, 2011.

27. Neugebauer, R., Drossel, W., Wertheim, R., Hochmuth, C., and Dix, M., "Resource and Energy Efficiency in Machining using HighPerformance and Hybrid Processes," Procedia CIRP, Vol. 1, pp. 316, 2012.

28. Diaz, N., Helu, M., Jarvis, A., Tönissen, S., Dornfeld, D., et al., "Strategies for Minimum Energy Operation for Precision Machining," Laboratory for Manufacturing and Sustainability, 2009.

29. Vijayaraghavan, A. and Dornfeld, D., "Automated Energy Monitoring of Machine Tools," CIRP Annals-Manufacturing Technology, Vol. 59, No. 1, pp. 21-24, 2010.

30. Devoldere, T., Dewulf, W., Deprez, W., Willems, B., and Duflou, J., "Improvement Potential for Energy Consumption in Discrete Part Production Machines," in: Takata, S. and Umeda, Y., (Eds.), Advances in Life Cycle Engineering for Sustainable Manufacturing Businesses, Springer, London, pp. 311-316, 2007.

31. Rajemi, M., Mativenga, P., and Aramcharoen, A., "Sustainable Machining: Selection of Optimum Turning Conditions based on Minimum Energy Considerations," Journal of Cleaner Production, Vol. 18, No. 10, pp. 1059-1065, 2010.

32. Helu, M., Behmann, B., Meier, H., Dornfeld, D., Lanza, G., et al., "Impact of Green Machining Strategies on Achieved Surface Quality,” CIRP Annals-Manufacturing Technology, Vol. 61, No. 1, pp. 55-58, 2012.

33. Sato, R., "Mathematical Model of a CNC Rotary Table Driven by a Worm Gear," International Journal of Intelligent Mechatronics and Robotics, Vol. 2, No. 4, pp. 27-40, 2012.

34. Kong, D., Choi, S., Yasui, Y., Pavanaskar, S., Dornfeld, D., et al., "Software-Based Tool Path Evaluation for Environmental Sustainability," Journal of Manufacturing Systems, Vol. 30, No. 4, pp. 241-247, 2011.

35. Humphrey, S., Papadopoulos, H., Linke, B., Maiyya, S., Vijayaraghavan, A., et al., "Power Measurement for Sustainable High-Performance Manufacturing Processes," Proc. of the CIRP International Conference on High Performance Cutting, HPC2014, Procedia CIRP, Vol. 14, pp. 466-471, 2014.

36. Rangarajan, A. and Dornfeld, D., "Efficient Tool Paths and Part Orientation for Face Milling," CIRP Annals-Manufacturing Technology, Vol. 53, No. 1, pp. 73-76, 2004.
37. Campatelli, G. and Scippa, A., "Prediction of Milling Cutting Force Coefficients for Aluminum 6082-T4," Proc. of the CIRP International Conference on High Performance Cutting, HPC2012, Procedia CIRP, Vol. 1, pp. 563-568, 2012. 